\section{The omohyoid and sternoclei- domastoid muscles entrapment of the internal jugular vein: Which role in Mèniére disease patients? Treatment perspective description}

\author{
Davide Piraino, ${ }^{1}$ Girolamo Garofalo, ${ }^{2}$ \\ Antonella Faletra, ${ }^{2}$ Aldo Messina $^{2}$ \\ ${ }^{1}$ Interventional Cardiology Unit; \\ ${ }^{2}$ Audiology Section, P. Giaccone \\ Universitary Hospital, Palermo, Italy
}

\begin{abstract}
The objectives were to analyze the Internal Jugular vein entrapment caused by muscles compression and the possible role and correlation in Mèniére disease. We describe the eco-color Doppler evaluation of a sternocleidomastoid and omohyoid muscles compression of internal jugular vein in a Mèniére patient, responsible of an anomalous venous cerebral and ear outflow. The proposed treatment was a three months muscolar decontractration physiotherapy program. The physiotherapy session allowed a complete muscles relaxation with an improvement of Internal Jugular vein caliber associated to a normalized cerebral and inner ear venous outflow and a progressive attenuation of Mèniére symptoms during the treatment and its disappearance at the end of the physiotherapy program. The comfort of the patient was confirmed during one-year follow-up. Muscles entrapment of Internal Jugular veins may be correlated with an anomalous cerebral and inner ear venous outflow, promoting the Mèniére disease symptoms. Physiotherapy treatment may represent an intriguing option alternatively of muscle surgical. The present case seems to indicate a possible first line treatment by physiotherapy, reserving surgical resection to not responders. Further studies with a wider sample of patients are warranted.
\end{abstract}

\section{Introduction}

Zamboni and his team have first investigated the role of venous system in the cerebral hemodynamic mechanisms as well as brain and ear physiology, describing its characteristics through the anatomical and functional point of view, indicating the differences in terms of elasticity and compliance $^{1}$ and underlining the comparable importance in relation to the arterial system that, until then, had attracted almost completely the attentions and finally defining the chronic cerebro-spinal venous insufficiency (CCSVI) as an altered head outflow due to intraluminal defects ${ }^{2}$ and generating an increased interest in the last years about the possible involvement as common ethiopategenetic mechanism in different pathologies as multiple sclerosis (MS), Parkinson or Alzheimer disease, Mèniére syndrome (MèS) and migraine. ${ }^{3}$

Afterward the progress made in the evaluation of possible anomalies able to effect an altered cerebral outflow, allowed to understand how, in addition to the detection of vascular defects described as Truncular venous malformations (TVMs) and expressed as the result of vascular trunk developmental defects occurring during the embryogenesis later stage, the point of view has progressively widened, evaluating the possible role of the presence of a muscular external compression. ${ }^{4}$

Recently, Mandolesi et al. reported a classification of CCSVI in three different patterns: ${ }^{5}$ i) type 1 CCSVI due to an endovascular obstacle, called hydraulic; ii) type 2 CCSVI due to a muscular compression without endovascular anomalies, called mechanical; iii) type 3 CCSVI presenting both endovascular and extravascular anomalies, called mixed; iv) all these three types determinate an anomalous outflow drenage.

We report a case of internal jugular vein (IJV) entrapment by Omohyoid and Sternocleidomasoid muscles (OM and SCM respectively) in a patient affected by MèS, as well as a new possible treatment option. Informed consent was provided by the patient.

\section{Case Report}

A 54-years-old woman with MèS disease related to fastidious and continuous bilateral tinnitus associated to auricular fullness (mainly in the left ear) and weekly episodes of dizziness related sometimes to autonomic phenomena as nausea and vomiting was admitted to our Audiology Department and was subjected to audiometric examination (normal) as well as cerebral computed tomography and magnetic resonance imaging scans (negative). Ethics board approval was obtained for this study. We decided to perform a cerebrospinal outflow Eco-color Doppler (ECD) ultrasound evaluation of the vertebral vein (VV) and IJV, revealing a partial muscular compression of right IJV and a complete muscular compression of left IJV in J2 and J3 segments (Figure 1A and B) responsible of an anomalous venous flow, in
Correspondence: Davide Piraino, Interventional Cardiology Unit, University Hospital P. Giaccone, via del Vespro n. 128, Palermo, Italy.

Tel./Fax: +39.091.6554312

E-mail: pirainod@libero.it

Key words: Internal jugular vein; muscle entrapment; physiotherapy treatment.

Conflict of interest: the authors declare no potential conflict of interest.

Received for publication: 12 August 2018.

Revision received: 23 September 2018.

Accepted for publication: 4 September 2018

This work is licensed under a Creative Commons Attribution 4.0 License (by-nc 4.0).

(C) Copyright D. Piraino et al., 2018

Licensee PAGEPress, Italy

Veins and Lymphatics 2018; 7:7760

doi:10.4081/vl.2018.7760

almost complete resolution during Valsalva maneuver, revealing the real caliber of IJV and the absence of vascular anomalies with a normal outflow, denoting a type 2 of CCSVI.

Therefore we directed the patient to a physiotherapy treatment for muscular compression mainly focused to SCM and OM (first used for this CCSVI type), with the goal of a muscle complete relaxation and a restoration of physiological IJV caliber and venous outflow. After one month treatment ECD analysis revealed a partial muscle decompression and partial caliber improvement of IJV (Figure 1C and D) while after a complete three months cycle (Table 1) of physiotherapy treatment, we repeated ECD analysis observing an almost complete basal reduction of muscle compression and a normalized outflow through IJVs (Figure $1 \mathrm{E}$ and F) with an enhance of comfort for the patient due to tinnitus progressive attenuation and auricular fullness disappearance. During one-year follow-up, the patient maintained the improved quality of life, without no more episodes of dizziness.

\section{Discussion}

Mèniére disease was described for the first time in 1861 and defined as an idiopathic syndrome of endolymphatic hydrops, ${ }^{6}$ but still today the mechanism of this pathology is not yet fully understood and several heterogeneous factors are related as possible responsibles: inflammation or autoimmune disease, genetic predisposition, 
hypoproteinemia, viral infection, dietary deficiencies. $^{7}$

In the last years, the presence of cerebral venous system abnormalities encountered by ECD, according to the CCSVI criteria and originally exclusively related as a contributing factor to $\mathrm{MS},{ }^{2}$ with a significant impact on brain pathophysiology, especially on intracranial fluid balance and cerebrospinal fluid (CSF), ${ }^{8}$ has been recently correlated with MèS, since these cerebrovascular alterations could also affect inner ear districts, which also drain into the IJV system. An anomalous venous outflow through the IJV and VV may be responsible of a progressive reduction or altered venous drainage into the venous cerebrospinal system of the anterior and posterior vestibular veins and/or of the cochlear veins with the result of an excess of endolymphatic volume secondary to stria vascularis cellular damage loosing the normal homeostasis. The elevation of hydrostatic pressure, due to venous outflow obstruction, the chronic venous stasis and the damage of stria vascularis increase the fluid from the capillaries into the endolymphatic space in a mechanism involved in MèS. ${ }^{9}$

Recently, Bavera et al. assessed the presence of cerebral outflow anomalies in both, a population affected by MS and a population affected by Mès, demonstrating that not only there was a high prevalence of these anomalies in these two populations compared to a control population, ${ }^{10}$ but also and above all that there were different patterns in the two pathologies.

Their work underlines that CCSVI can be considered as a common mechanism, which differs as a pattern according to the pathology of MS or Mès, observing in MS patients anomalies mainly related to the $\mathrm{J} 1$ and $\mathrm{J} 2$ segments, compared to the population with Mès, which would present more anomalies in the J2-J3 segments, as well as jugular bulb anomalies and mediolateral or anteroposterior position that may determining encroachment of the surrounding structures.

Our team has first reported the treatment of a type 1 CCSVI, the so-called hydraulic form, due to venous anomalous outflow related to vascular obstacle in a MèS patient $^{11}$ confirmed by phlebography and treated through IJV angioplasty.

In case of Type 2 CCSVI, the called mechanical form, due to muscles compression of IJV, the only described treatment in literature is a surgical procedure through an OM surgical transection together with a patch angioplasty. ${ }^{12}$

In a population of 56 patients affected by CCSVI, Zamboni et al. showed interesting results by combining to the procedure of endophlebectomy with removal of the defective valves (used as an alternative to the IJV angioplasty), the muscular resection, in cases where a muscular compression on the IJV or VV was present. In this article Zamboni showed how endophlebectomy could be a viable alternative to angioplasty (because of IJV angioplasty is burdened by recurrences of anomal outflow, especially in cases of nonmobile valve leaflets), associating muscular resection, in the course of the intervention, in case of pattern III CCSVI (the mixed form due to vascular anomalies and muscle compression), reestablishing a complete normalization of cerebral outflow. ${ }^{13}$

Here we describe first the treatment of a MèS patient with IJV entrapment by SCM and $\mathrm{OM}$ and anomalous venous outflow with a not invasive option. The physiotherapy treatment has allowed a progressive muscles decontraction and a directly proportional IJV caliber improvement, avoiding a surgical intervention. ECD analysis performed both during the treatment and at the end of a three months cycle, confirmed the normalized venous outflow and the patient improvement quality of life, confirmed during one-year follow-up.

The conservative treatment, represented by a purposeful decontracting physiotherapy of the OM and SCM muscles (as described in Table 1) and their compression exerted on the IJV, may represent the first-line treatment for such patients, because clearly less bloody than muscular resection, the only treatment so far available.

The surgical option, clearly, can be a second choice treatment in case the targeted physiotherapy is not able to resolve the entrapment of the IJV.

The present case seems to indicate a possible first line treatment by physiotherapy, reserving surgical resection to not responders. Further studies with a wider sample of patients are warranted.

Table 1. SCM and OM muscles physiotherapy treatment.

\section{Physiotherapy treatment for muscular compression}

Physiotherapy and osteopatical approach, releasing vertebrae and bone fragments where these muscles are attached, giving it back their proper functionality, just as much as to relevant metameres innervation

Fascial approach starting from the myofascial tissue shall be carried out manoeuvring of pompage, overstretching and mild compression of the muscle tissues until a feeling of their re-lease

Both approaches should be implemented with repositioning methods on the planes (sag-ittal, coronal, transverse)

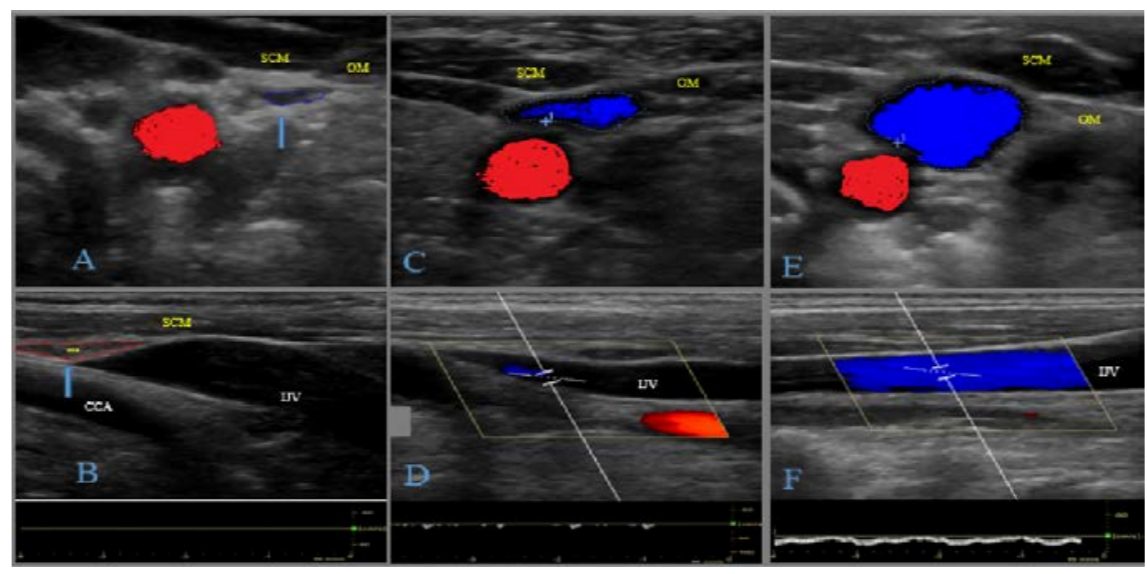

Figura 1. A) SCM and OM compression of IJV (blue arrow) during trasversal ECD analysis. The IJV appears oval, loosing its natural shape. B) Longitudinal ECD analysis with evidence of IJV entrapment (blue arrow) and the absence of flow at PW Doppler. C) Partial muscles relaxation and IJV caliber improvement after one month physiother-apy treatment: longitudinal ECD analysis confirmed the partial muscle compression resolution with a minimal flow detected at PW Doppler (D). E) Complete IJV caliber improvement at the end of physiotherapy treatment, confirmed in longitudinal axis and a normalized outflow in PW Doppler (F). 


\section{8;7:7367.}

\section{Conclusions}

The recovery of cerebral venous haemodynamics and inner ear concurrent to a progressive improvement of the patient conditions and to the desappearance of MèS symptoms raises different matters: i) what role can we actually give to the muscular components and to the compression that they exert altering and impairing the venous outflow; ii) if the variability of muscolar tone could be related in a meaningful way to a more or less correct changing of the posture held by the patience, to his job, to his daily physical activities, so acting on the venous aspect a fairly substantial and decisive compression; iii) if this muscular compression variabilty could be reflected to the typical variability and fluctuations of symptoms of MèS patients, representing the physiotherapy treatment a valid option with effective benefits, rather than the surgical muscle resection.

\section{References}

1. Zamboni P, Tavoni V, Sisini F, et al. Venous compliance and clinical implications. Veins and Lymphatics
2. Zamboni P, Galeotti R, Menegatti E, et al. Chronic cerebrospinal venous insufficiency in patients with multiple sclerosis. J Neurol Neurosurg Psychiatry 2009;80:392-9.

3. Zamboni P. Why Current Doppler Ultrasound Methodology Is Inaccurate in Assessing Cerebral Venous Return: The Alternative of the Ultrasonic Jugular Venous Pulse. Behav Neurol 2016; 7082856.

4. Zamboni P. How to objectively assess jugular primary venous obstruction. Veins and Lymphatics 2014;3:4195.

5. Mandolesi S, D'Alessandro A, Ciccone $\mathrm{MM}$, et al. Analysis of patients with chronic cerebro-spinal venous insufficiency and multiple sclerosis: identification of parameters of clinical severity. Veins and Lymphatics 2015;4:4570.

6. Lopez-Escamez JA, Carey J, Chung $\mathrm{WH}$, et al. Diagnostic criteria for Meniere's disease. J Vestib Res 2015;25:1-7.

7. Pirodda A, Brandolini C, Raimondi MC, et al. Meniere's disease: update of etiopathogenetic theories and proposal of a possible model of explanation. Acta Clin Belg 2010;65:170-5.
8. Zamboni P, Menegatti E, Occhionorelli $\mathrm{S}$, Salvi F. The controversy on chronic cerebrospinal venous insufficiency. Veins and Lymphatics 2013;2:e14.

9. Filipo R, Ciciarello F, Attanasio G, et al. Chronic cerebrospinal venous insufficiency in patients with Ménière's disease. Eur Arch Otorhinolaryngol 2015;272:77-82.

10. Bavera PM, Di Berardino F, Cecconi P, et al. Chronic cerebro-spinal insufficiency in multiple sclerosis and meniere disease: same background, different patterns? Veins and Lymphatics 2017;6:6533.

11. Piraino D, Andolina G, Messina A. Truncular Venous Malformations in Ménière Disease Patients: The Key for a New Treatment Perspective? J Vasc Interv Radiol 2016;27:766-8.

12. Gianesini S, Menegatti E, Mascoli F, et al. The omohyoid muscle entrapment of the internal jugular vein. A still unclear pathogenetic mechanism. Phlebology 2014;29:632-35.

13. Zamboni P, Menegatti E, Cittanti C, et al. Fixing the jugular flow reduces ventricle volume and improves brain perfusion. J Vasc Surg Venous Lym Dis 2016;4:434-45. 